Spinal Cord (1997) 35, 869-871

\title{
Author index to volume 35
}

Key to abbreviations:

(BR) Book review; (E) Editorial; (E) Editorial Envoi; (L) Letter; (O) Obituary notice; (R) Review

Abel R 443

Açikgöz B 189 (L)

Adkins R 282

Agranov E 48, 850

Agrò EF 37

Akatsu T 718

Aki S 386

Aldinger W 446

Allatt RD 121

Allison GT 217

Almenoff PL 652

Alpdogan TB 704

Amy S 694

Andersen JL 1

Anderson J 282

Apple D 264 (E)

Asbeck FWA van 109

Ashworth B 64

Ataoğlu S 531

Athanasou J 238

Aung TS 147

Baba H 26, 163, 632, 674, 710

$$
\text { (L), } 725
$$

Bach J 805

Badiali D 116

Baert L 613

Balderston RE 358

Bangsbo J 1

Banovac K 158

Barnardi M 383

Barros TEP 358, 641

Bauman WA 652

Bayik M 704

Bell G 86

Benko H 540

Bennett CJ 282

Benzel EC 599

Berhammer A 493

Berker E 386

Biering-Sørensen F 1, 76, 192 , $193(\mathrm{O}), 641,862$

Binard J 613

Bingley J 558 (L), 608

Blackmer J 245

Blanch L 574

Blomstrand C 17

Blumhardt LD 361

Böhm V 443

Bötel U 40, 420

Bouchier AD 786 (BR)

Boudaoud L 151

Bourgeois I 546

Bracci F 116
Bracken MB 266

Brigden K 574

Brønnum-Hansen H 76, 862

Brook GA 474

Brösamle C 469

Broton JG 292

Brouwer B 205

Brown CW 710 (L)

Brown DJ 238, 275, 658, 805

Burnham RS 86, 647

Burr RG 521

Bussel B 151

Campus G 33

Canupp KC 314

Carroll CM 341

Castellano V 37, 58, 116, 383, 516

Castello T 664

Catz A 48, 850

Celikel CA 704

Chancellor MB 546

Chappel R 710 (L)

Charlifue S 320, 349, 608

Chen Y-C 550

Chiba M 773

Chuang C-C 841

Clark PF 121

Clarridge JE 534

Clevenger FW 599

Clifton GL 190 (L)

Climstein M 680

Clinchot DM 181, 256

Cohen ME 308

Colachis III SC 181, 256

Cooper D 608

Corazziari E 116

Corey P 818

Cousins MJ 69, 526

Creasey G 266

Crewalk J-A 546

Cunningham DA 121

Curt A 368

Dallmeijer AJ 104

Darouiche RO 534

Davis GM 580, 680

De Ridder D 613

Deftos LJ 223, 374

Demirel G 22

DeVivo MJ 314, 509, 809

Di Lucente L 516

Diaz F 282

Dietz V 368
Dijk AJ van 109

Dijkers M 829

Ditunno Jr JF 266, 275

Dizien O 151

Dollfus P 187, 275, 785 (BR), 803

Domínguez R 664

Donovan WH 266, 275, 565

Döşoğlu M 22

Doughan AM 253

Drouet L 151

Ducker TB 266

Dutton J 320, 349

Dzidic I 620

El-Masry WS 147, 358, 798, $861(\mathrm{~L})$

Elbași N 22

Endo H 554

Engel S 580

Erdoğan F 531

Erjavec T 540

Eskesen B (deceased) $193(\mathrm{O})$

Exner G 410 (E), 411, 415

Fahey A 680

Felici F 383

Festvåg LV 503

Filiz M 386

Flogering HTM 104

Foley SJ 96

Föllinger S 446

Foreman P 238

Frankel H 275, 335, 796, 814

Frisbie JH 303

Fritts K 286

Fromberg G 437

Fujimura Y 729, 752, 777

Fuoco U 58, 116

Furusawa N 632, 674, 725

Galbo $\mathrm{H} 1$

Galloway A 198 (R), 709 (L)

Ganuza FJR 124

Garau A 33

Garber SL 266

García-Fernández L 664

Gardner B 814

Gefen JY 308

Gelmann AS 308

Gerner H-J 410 (E), 443, 446

Giannantoni A 37

Giral A 704

Giuffre JM 286
Gläser E 40, 420

Glass CA 320, 349

Goepel M 456

Goldsmith HS 190 (L)

Gonzalez, F 158

Gorman PH 569

Gounden P 43

Gramm M 493

Graves DE 171

Green BA 292

Grimm DR 652

Gruenthal M 686

Grüninger W 446

Guest R 292

Gurmen N 704

Gurwitz JH 394

Gutteridge G 805

Haas B 64 (L)

Habib FI 116

Hackbusch B 487

Hamilton LR 617

Harari D 394

Harris P 135 (E), 259 (BR), 265 (E), 786 (BR), 793 (EE), 802, 804, 865

Harrison R 129 (BR)

Hartkopp A 76, 862

Harvey LA 580

Hashimoto S 757

Hashizume Y 740

Hatsuyama Y 720

Hattar EZ 253

Hayes KC 121

Henmi T 554

Herbison GJ 308

Hermann-Gruber, M 487

Heydrick D 569

Hill ST 658

Hong RD 546

Hopkins-Rosseel DH 205

Hopman MTE 104

Hoshi N 773

Hoshino Y 735

Howard L 605

Hsu RW-W 550

Huang T-J 550

Ide M 234

Ido K 183

Ikata T 717 (E)

Illis LS 258 (BR), 259 (BR)

Imasato H 757

Imura S 632, 674 
Inoue A 757

Ishihara H 763

Itzkovich M 850

Iwatsubo E 498, 718

Jackson HF 320, 349, 694

Jacobs PL 292

Jaeger RJ 299

Janzen J 861 (L)

Jaspers P 585

Jayakumar PN 624

Jonas HP 487

Kabins MB 286

Kakar A 629

Kannisto M 747

Kao M-J 841

Karacan I 22, 531

Karamehmetoğlu SS 22, 531

Karan A 386

Karčnik T 540

Kaspar A 176

Kawahara H 725

Kawahara N 26, 163, 710 (L)

Kawamura K 590, 595

Kawar JS 253

Kennedy P 617, 814

Kim KT 690

Kimura T 720

Kinoshita H 857

Kishi S 740

Kitagawa H 763

Kjaer M 1

Klose KJ 292

Kluger P 429

Ko H-Y 690

Kobayashi M 234

Kodalli N 704

Kojima A 768

Kokubo Y 632, 725

Komisaruk BR 136

Komori H 757

Korge A 429

Kornhuber HH 176

Kotani S 183

Koyama K 768

Koyuncu H 22, 531

Kralj A 540

Kramer G 456, 463

Krishnan KR 403 (L), 557 (L), $558(\mathrm{~L}), 709(\mathrm{~L})$

Kruse D 456

Kurien T 99

La Banda F 124

Lask D 48

Lenger R 92

Lesser M 652

Liao Y-S 550

Lien I-N 841

Lim TC 658

Lin K-H 841

Lind B 17

Lissens MA 402 (L)

Löchner-Ernst D 456, 463

Lortat-Jacob S 151
Lundqvist C 17

Luttwak ZP 48

Macaluso A 383

MacArthur D 121

McCary A 282

McClelland J 526

McColl MA 818

McDicken I 709 (L)

McFarlane JP 96

McGlinchey-Berroth G 394

MacLean I 86

Madan VS 629

Madersbacher HG 498

Maezawa Y 632, 674, 725

Maisch U 176

Malone LA 647

Mandalka B 463

Marchettoni P 383

Marino RJ 266

Marks J 800

Marrosu MG 33

Marshall S 245

Martin EME 223, 374

Martin T 86

Matias B 282

Matsui H 763

Matsumoto M 777

Matthews JM 647

Mattson-Prince J 326

Mauch E 176

Maynard Jr FM 266

Mazaira J 124

Meindl R 40

Meinecke F-W 410 (E), 411 , 415

Meiners T 443

Mello MT 248

Menter RR 608, 805

Metzmacher K 446

Meyer Jr PR 358

Middleton JW 574

Midha M 332

Mimatsu K 740

Minaker KL 394

Mitzukami M 720

Mizuno K 163, 710 (L)

Mochida J 700

Mohr T 1, 192

Momose H 179

Montalvo R 124

Monteiro AA 641

Montgomerie JZ 282

Moore AP 361

Moore K 332

Moslavac S 620

Mueller M 171, 686

Mukamel E 48

Murphy G 238

Müslümanoğlu L 386

Nacimiento W 474

Nagata K 757

Nakajima A 768

Nakamura K 735

Nakamura M 729, 752, 777
Nakamura R 720

Nas K 531

Needham-Shropshire B 292

New PW 658

Newton-John T 580

Niedeggen A 40, 420

Nihei R 720

Nishi Y 777

Noriki S 632

Noth J 474

Nuseibeh I 335, 521, 641, 814

Obreza P 540

Ogata H 234, 590, 595

Ohnari H 26

Okada K 773

Okada Y 183, 710 (L)

Okuyama K 773

Olona M 664

Olson WH 171, 686

Olson WL 686

Oota K 700

Orritt C 320, 349

Otom AS 253

Öztürk Y 386

Pace A 58

Paeslack V 446

Paraira M 664

Parthemore JG 223, 374

Pau AC 33

Paulus C 176

Paz A 48

Peckham PH 569

Peeraer L 585

Pentelenyi TJ 358

Pentland B 258 (BR)

Perkash I 498

Perre G van der 585

Pesce F 37

Petegem W van 585

Peterson K 574

Petrelli A 516

Pischedda MP 33

Poppel H van 613

Porru D 33

Post MWM 109

Poyares D 248

Prakash V 629

Priebe MM 171, 534, 686

Puet TA 694

Ramírez L 664

Ramón S 664

Raymond J 680

Reid G 605

Renganathan R 99

Richards JS 314

Ring H 850

Rivas DA 546

Rodic B 368

Rodio A 383

Rodriguez DJ 599

Ronen J 48

Rösche J 176

Rosenberg N 92, 861 (L)
Roussi J 151

Rübben H 456

Rueda AD 248

Ruidisch M-H 437

Sairyo K 554

Sarac AJ 531

Sarkarati M 394

Sartoris DJ 223, 374

Sato K 773

Scarpa RM 33

Scharf H-P 429

Schmidt AB 437

Schmidt RR 308

Schmitt-Dannert H-H 493

Schrijvers AJP 109

Schurch B 368

Schwab ME 469

Scivoletto G 58, 116, 516

Seidenschnur A-M 76, 862

Sekar P 509

Sellar R 785 (BR)

Sett P 709 (L)

Shafik A 53

Shah PJR 96

Shenaq SM 641

Sherwood AM 171, 686

Shi J 390

Shiba K 718

Shibasaki K 641

Shibata M 700

Shih H-N 550

Shimizu K 183

Shpaser R 48

Shterenshis, MV 139, 711 (L)

Siddall PJ 69, 526

Silva AC 248

Silver J 402 (L), 711 (L)

Singer KP 217

Sintonen H 747

Siösteen A 17

Smith MB 580

Smith WDF 121

Snoek G 104

Soni BM 403 (L), 558 (L), 709 (L)

Sørensen JL 641

Sorgia M 33

Soy D 386

Spinici G 33

Spungen AM 652

Srikanth SG 624

Stahr G 487

Stanghelle JK 503

Steadward RD 86, 647

Steele DJR 303

Stein H 92, 861 (L)

Stein R 86

Stephenson TP 498

Stirling P 818

Stöhrer M 456, 463, 498, 859

Störmer S 446

Stover SL 129 (BR), 266

Stroh Wuolle K 569

Strubreither W 487

Sullivan L 17, 229 
Sullivan M 17

Sumiya T 590, 595

Suranjan B 99

Sutton JR 680

Suyama T 720

Sved P 526

Szollar SM 223, 374

Taheri A-S 474

Takechi H 590, 595

Takemitsu Y 718

Taktak DM 61

Tamburro F 37

Tamir A 48, 850

Tanaka S 26

Tang HH 390

Tator CH 266

Taylor DA 69

Telander M 229

Thalgott JS 286

Timlin M 286

Tobimatsu Y 720

Toh E 700
Tokuhiro A 590, 595

Tomita K 26, 163, 710 (L)

Toprak A 704

Toribatake Y 26, 163, 710 (L)

Tsauo J-Y 841

Tsuji H 763

Tufik S 248

Turba RM 299

Uchida K 632, 674, 725

Ueta $\mathrm{T} 718$

Urayama K 735

Usai E 33

Vaidyanathan S 191 (L), 403

(L), 557 (L), $558(\mathrm{~L}), 709$ (L)

Vare V 574

Vasudev MK 624

Vaughan GT 786 (BR)

Vespasiani G 37

Vogler L 493

Vona VU 58
Vuong PN 861 (L)

Wada F 234

Wada M 674

Wagner A 1

Waites KB 314

Walker J 818

Walker N 446

Waller L 229

Walsh EG 64 (L)

Wang AY 299

Watanabe M 729, 752, 777

Watanabe T 546

Waters RL 266

Watt J 805

Weisz I 92

Weitzenkamp D 608

Wheeler GD 647

Whipple B 136

Whiteneck G 608

Wienke Ch 446

Wilberger JE 266

Wilkins R 818
Willems J 710 (L)

Woodhams PL 474

Woude LHV van der 104

Wyndaela JJ 213

Yabe Y 729

Yamada K 179

Yamada S 700

Yamamoto M 179

Yano H 720

Yarkony GM 299

Yato Y 729, 752

Yeo JD 574

Yonezawa T 763

Young A 238

Young M 282

Young W 266

Yu J 390

Zhou GC 390

Zigler JF 358

Zimmermann M 446

Zupan A 540 